\title{
The Role of Approach and Avoidance Motivation and Emotion Regulation in Coping Via Health Information Seeking
}

\author{
Anita Chasiotis ${ }^{1}$ (D) $\cdot$ Oliver Wedderhoff $^{1} \cdot$ Tom Rosman $^{1} \cdot$ Anne-Kathrin Mayer $^{1}$ \\ Published online: 26 October 2019 \\ (C) The Author(s) 2019
}

\begin{abstract}
When dealing with a health threat, health information seeking (HIS) is a prominent way of engagement coping. Yet, there is only limited research as to its motivational and emotion regulatory antecedents. We present a theoretical model integrating approach and avoidance motivation, emotion regulation, HIS self-efficacy, and problem and emotion coping focus as predictors of HIS. We propose that, in the context of HIS, (1) approach and avoidance motivation have a direct effect on emotion regulation ability (positive and negative, respectively), (2) approach and avoidance motivation have indirect effects on intended comprehensiveness of search via emotion regulation, HIS self-efficacy and problem coping focus, (3) avoidance motivation has a direct effect on emotion coping focus. Our model was tested by means of structural equation modeling in a sample of university students $(N=$ 283). Model fit was good, and all three hypotheses were supported. We show that emotion regulation ability is essential to explain the effects of approach and avoidance motivation on HIS as it fosters self-efficacy and a problem coping focus. The direct effect of avoidance motivation on emotion focus may represent an alternative way of coping with a health threat for those individuals who are highly sensitive to threat-related emotions.
\end{abstract}

Keywords health behavior $\cdot$ coping $\cdot$ emotion regulation $\cdot$ self-efficacy $\cdot$ structural equation modeling

Experiencing a health threat challenges individuals in two ways. Not only do they have to cope with the health problem itself. They are also required to deal with aversive emotions like anxiety and worry. These essential processes in dealing with threatening situations have been referred to as emotion and problem focused coping (e.g., Lazarus 2006). With regard to these two basic ways of coping, other authors have further distinguished between engagement coping and disengagement coping (Carver and Connor-Smith 2010). Disengagement coping encompasses a diverse set of strategies to deal with a threat and with resulting aversive emotions by avoiding and denying the threat as a whole (e.g., Derakshan et al. 2007). Engagement coping, in contrast, is characterized by actively facing up to the threat or the threat-related aversive emotions. When facing a health threat, a prominent engagement coping strategy which can be pursued with a problem or emotion focus, is health information seeking (Shiloh and

Anita Chasiotis

ac@leibniz-psychology.org

1 Leibniz Institute for Psychology Information, Universitaetsring 15, 54296 Trier, Germany
Orgler-Shoob 2006). We define health information seeking (HIS) as actively and deliberately searching and retrieving health related information, e.g. about prevention, symptoms and diseases using any kind of information channels. Hence, not only searching the internet for information about recently occurred symptoms, but also visiting the family doctor for a check-up or asking friends and colleagues are possible ways of HIS.

Helping individuals to adequately cope with a (health) threatening situation via information seeking by considering their needs and preferences requires research focusing on motivational and affective dispositions which impact coping behavior (Lazarus 2006). Past research has identified motivational and affective dispositions as most pertinent in explaining interindividual differences in HIS (Gerend and Shepherd 2007; Hastall and Wagner 2017; Hevey and Dolan 2014; Van't Riet and Ruiter 2013). For example, in an experimental study, Hastall and Wagner (2017) found that lowanxious individuals who were highly avoidance-motivated selected more loss-framed (compared to gain-framed) health information articles for reading. Furthermore, in a comprehensive literature review, van't Riet and Ruiter (2013) point out that differing levels of emotion regulation ability affect the exposure to health-promoting information. In the present 
study, we strive for a better understanding of how these dispositions impact HIS via simultaneously identifying relevant mediators and unique modes of effects. This is, on the one hand, fruitful for future research on the relationship between personality and health information behavior. On the other hand, scrutinizing the impact of these dispositions on HIS provides clinical practitioners with professionally relevant insights concerning interindividually differing information needs of their patients.

In past studies, there have been valuable approaches to capture individual differences in engagement and disengagement coping styles with regard to information seeking under a (health) threat (e.g., vigilance vs. cognitive avoidance, Krohne and Hock 2011; monitoring vs. blunting, Miller 1987; sensitizing vs. repressing, Byrne 1961). These concepts of coping styles essentially capture behavioral tendencies that are mainly attributed to dispositional preferences (Krohne and Hock 2011). According to these and similar conceptualizations of coping styles, the dispositional preference to cope with a threat via avoiding and neglecting the threat itself, for example, would lead to behavioral avoidance and neglect of threat-relevant information (as is the case with so called "blunters" or "repressors"). Correspondingly, the main focus of this research was to investigate individual differences in two opposing, dichotomously conceptualized coping styles: (1) in actively seeking (e.g., monitoring) or (2) in avoiding (e.g., blunting) potentially threatening information. If potentially threatening information is not avoided and thus, an active search for information is conducted, it can vary on a wide continuum with regard to its comprehensiveness. Individuals may conduct an exhaustive search, taking into account many perspectives and facets of a topic. They may also, however, conduct a rather shallow or biased search which is mainly driven by the motivation to find reassuring or confirming (rather than accurate) information (Hart et al. 2009). Thus, research on HIS exclusively focusing on interindividual differences in choosing between all-or-none alternatives (avoiding vs. seeking) neglects possible variations in the realization of an active search. Up to now, only little is known about the personality factors that are responsible for these interindividual differences in actively seeking health information (Litman and Lunsford 2010). Shedding light on such factors, however, is crucial for future research on interventions to help patients meet their information needs and become more responsible for their own health and wellbeing (Berkman et al. 2011). Existing research identified motivational and affective dispositions to be particularly relevant for HIS (see above). If we increase our knowledge with respect to the direct and mediated effects of these factors on HIS, corresponding interventions could be tailored to individuals differing in their motivational and affective dispositions. For example, individuals regularly experiencing difficulties in downregulating threat-related emotions such as anxiety may benefit from interventions that prevent them from confirming their fear through threat-directed searches (e.g., searching for "headache brain tumor" when suffering from a headache; White \& Horvitz, 2009). Furthermore, awareness in how far patients differ with regard to motivational and affective dispositions can aid clinical practitioners in following a needsensitive and flexible approach when providing health information (see also Kiesler and Auerbach 2006).

In the present study, we aim at developing and testing a theoretical model which for the first time integrates relations between motivational and emotion regulatory processes as well as emotion and problem foci in HIS. In the model, we also consider the differential impact of emotion focused vs. problem focused HIS on the intended comprehensiveness of the search (as a proxy for actual seeking behavior). Intended comprehensiveness is characterized by exhaustively seeking balanced information which may contradict one's own opinion (e.g., about vaccination). In the following section, we develop our final model (see Fig. 1) based on the existing evidence and derive specific hypotheses.

\section{Model Development}

As mentioned above, motivational dispositions play a crucial role when it comes to coping with threat. These motivational dispositions are generally understood of as basic, primarily genetically determined individual differences in motivation (Hartig and Moosbrugger 2003). Litman (2006) found that dispositional approach motivation, essentially capturing sensitivity to rewards and reward-related emotions like joy (Carver 2005), positively predicted engagement coping. Still, it remains unclear by which mechanisms this association is established. As for dispositional avoidance motivation, i.e., sensitivity to threat and threat-related emotions like anxiety, there were no associations with engagement coping. However, according to Carver (2005), there are essentially two ways in which avoidance motivation fosters action aiming at averting a threat. Reactive control refers to the avoidance of a threat by neglecting it, whereas effortful control refers to the avoidance of a threat by reducing or eliminating it. More specific, reactive control responds to danger via direct avoidance of the threatening cue. Here, the primary goal is to reduce anxiety and stress by evading the threat or the threatening information as a whole. Reactive control therefore serves a more emotionfocused and disengagement coping style. Effortful control, on the other hand, encompasses the initiation of approach behavior by superseding initial avoidance impulses, to serve a superordinate avoidance goal. This is in line with Showers and Boyce (2008) as well as Elliot and Church (1997) who postulate similar processes of motivated behavior. In the context of a health threat, this means that potentially threatening information is actively sought to help deal with the threat. For 


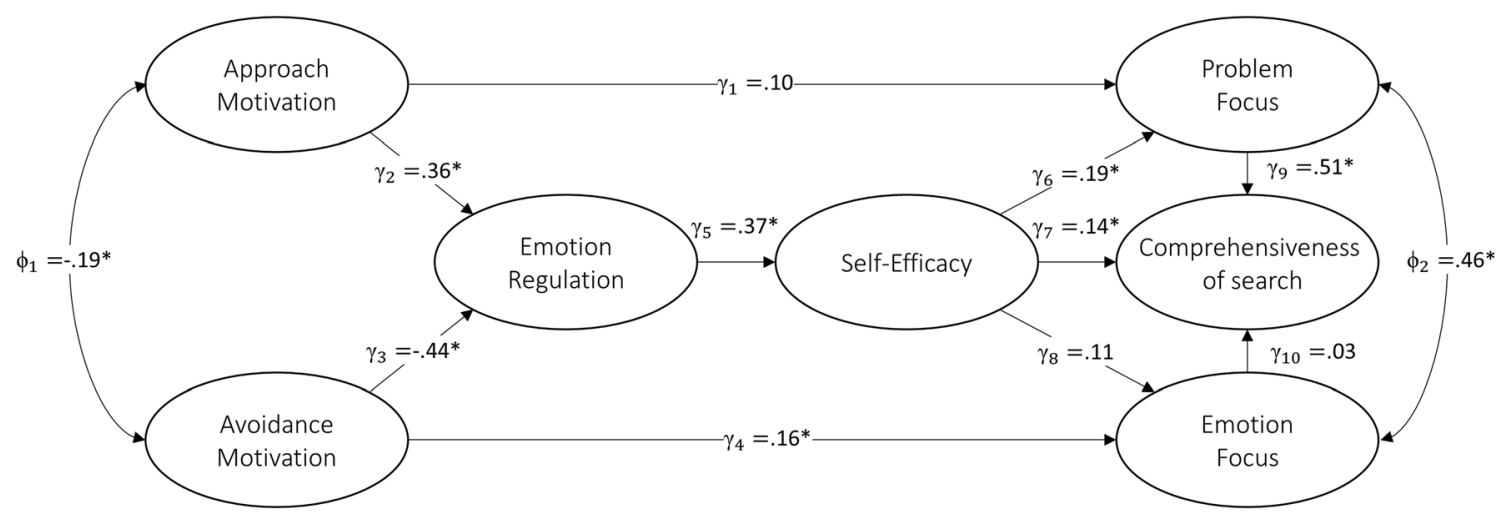

Fig. 1 Model including approach and avoidance motivation, emotion regulation, health information seeking self-efficacy and coping focus in health information seeking, and their effects on intended comprehensiveness of search. $* p<.05$

example, undertaking a cancer screening entails the immediate experience of inconvenient emotions like anxiety and worry, but essentially, early detection might increase chances of recovery. Threat prevention may thus serve both a problem focused and an emotion focused coping purpose as dealing with a threat directly also reduces anxiety in the long run (Folkman 2010). Thus, the lack of an association between avoidance motivation and engagement coping might be the result of two interfering mechanisms, with the underlying decisive conditions as to which mechanism will prevail being still largely unknown.

In considering the differential impact of approach and avoidance motivation on coping foci in HIS and the impact of these foci on intended information behavior (e.g., the intended comprehensiveness of a search), it is pertinent to take into account threat-related emotions like anxiety. In fact, such emotions may inhibit the initiation of higher order cognitive processes like effortful control (Carver 2005). Hence, in threatening situations, it might be necessary to downregulate these emotions, or to upregulate other, more adaptive emotions (e.g., worry instead of diffuse anxiety; Tamir 2016). As many researchers have pointed out, the concept of emotion regulation is to be distinguished from emotional sensitivity as it essentially captures the ability to leave or alter an emotional state, whereas the latter describes how quickly an emotional state is entered (Baumann and Kuhl 2002; Koole 2009; Tull et al. 2010). Thus, there is broad consensus in the literature that emotion regulation abilities are a precondition for the adaptation of a problem focused coping style (e.g., BlouinHudon et al. 2016; Das and Fennis 2008; Kuhl and Koole 2008). In fact, according to Kuhl and Koole (2008), the downregulation of negative affect enables a form of 'high level coping' via enhanced access to personal needs, values, and memory of past coping attempts (self-access). This enhanced self-access is essential in dealing with an existential threat. Despite a superordinate avoidance goal (threat prevention), self-access facilitates the initiation of adaptive approach behavior (e.g., via the additional upregulation of positive affect) to deal with the threat directly. Thus, a functioning emotion regulation ability facilitates the adaptation of problem focused coping. If, however, threat-related negative emotions cannot be dealt with sufficiently, less adaptive systems of personality dominate the coping process and facilitate behavioral avoidance and distraction processes. In this case, individuals would seek 'external' reassurance, with averting distress as their primary goal (Sweeny et al. 2010). This could mean, for example, that they reach out to other persons or engage in activities that allow them to find reassurance or mere distraction (both in contrast to actively addressing their health problem or unhealthy behavior; Van't Riet and Ruiter 2013).

It should be noted, however, that emotion regulation is not about completely eliminating feelings of discomfort or anxiety. Rather, it is about downregulating feelings of distress to a level where they are not detrimental to the initiation of adaptive behavior anymore, or can maybe even prove useful due to a resulting state of higher alertness (Braniecka et al. 2014; Tamir 2016). Accordingly, in their review of the literature, van't Riet and Ruiter (2013) point out that individuals, when confronted with threatening information, first have to get rid of potentially overwhelming negative feelings. Thus, they may, on the one hand, directly avoid dealing with the information and engage in distractive behavior, or simply deny its relevance. On the other hand, they may use more sophisticated emotion regulation strategies like cognitive reappraisal, which allow them to actively deal with the information by engaging in healthier behaviors. However, a prerequisite for this problem focused way of coping is the downregulation of negative emotions which facilitates actively dealing with a potential health threat (e.g. via engaging in a health behavior proposed by the respective piece of information). With respect to the relationship between approach and avoidance motivation and emotion regulation abilities, Tull et al. (2010) found that avoidance motivation was correlated positively with emotion regulation difficulties, whereas approach motivation was negatively associated with emotion regulation difficulties. A possible explanation for these results might be a motivation- 
specific 'affective vulnerability' (Dennis 2007; Tamir and Diener 2008): highly avoidance motivated individuals experience anxiety and negative emotions more often, which might lead them to the assumption that they are not very skilled when it comes to effective emotion regulation. On the other hand, highly approach motivated individuals experience excitement and joy more often and therefore may come to the conclusion that they are competent in dealing with (negative) emotions (Dennis 2007; Tamir and Diener 2008). Therefore, our first hypothesis is:

Hypothesis 1: Approach and avoidance motivation have a direct effect on emotion regulation abilities. This effect is positive for approach motivation and negative for avoidance motivation.

Until now, we have expected that approach and avoidance motivation predict emotion regulation. The latter, i.e. confidence in one's ability to downregulate threat-related distress, is known to predict general self-efficacy (Pocnet et al. 2017). Therefore, we would expect the same to be true for a more domain-specific form of self-efficacy, namely, self-efficacy related to HIS. Knowing that I will be capable of dealing with anxiety and stress during my search, I will be more convinced to find information I can use to deal with the health threat. Furthermore, as Rothermund (2011) points out, perceived controllability, which is strongly related to self-efficacy, fosters the uptake of a problem focus in information seeking. Confident in my ability to deal with and find the right health information for my purpose, I will find myself to be able to address my problem directly with the help of useful information. For this reason, we expect domain-specific self-efficacy to have an effect on problem focused information seeking which, in turn, should also have a positive effect on the intended comprehensiveness of search. Confident in my abilities to initiate a fruitful search, I may adapt a problem focus and with this, I aim at finding as much and preferably balanced information as possible (see also Litman and Lunsford 2010). Consequently, we expect approach and avoidance motivation to have an indirect effect on intended comprehensiveness of search via the interposed self-regulatory instances (i.e. emotion regulation and self-efficacy). Therefore, our second hypothesis is:

Hypothesis 2: There is an indirect effect of approach motivation as well as avoidance motivation on intended comprehensiveness of search via emotion regulation, HIS self-efficacy, and problem focused HIS.

As avoidance motivation, unlike approach motivation, captures sensitivity to threat and threat-related emotions like anxiety (Carver 2005), it can be supposed that individuals high in avoidance motivation are more prone to experience anxiety in a health threatening situation. One could thus expect these individuals to be in higher need of emotion regulation capacities compared to low-avoidance motivated individuals in the same threatening situation. A way to deal with threat-related emotions independent from and complementing individual emotion regulation ability, is with the help of external resources (i.e., health information), which may provide reassurance and calming (Litman and Lunsford 2010). In accordance with this, a vast body of evidence shows that dispositional avoidance motivation leads to a stronger emotion coping focus in various contexts, such as, for example, testing situations (Feil and Hasking 2008; Hasking 2006; Hundt et al. 2013; Schutz et al. 2008). For this reason, we expect avoidance motivation to positively predict an emotion focus in HIS. Therefore, our third hypothesis is:

\section{Hypothesis 3: There is a direct positive effect of avoid- ance motivation on emotion focused HIS.}

On the one hand, it is conceivable that an emotion focus in HIS leads to a short and rather superficial search that is discontinued as soon as any reassuring information is found. On the other hand, it could result in an extraordinarily extensive search (if one wants to be really sure). Owing to this ambiguity, the effect of an emotion focus in HIS on intended search comprehensiveness will be subject to an exploratory analysis.

The final linear structural model which we developed in accordance with these theoretical considerations and to test our hypotheses regarding specific direct and indirect effects is depicted in Fig. 1.

\section{Materials and Methods}

\section{Participants}

The study took place at a large university in Germany. Participants were recruited via student mailing lists, leaflets and flyers distributed on the campus, and direct acquisition during lectures. All participants agreed on an informed consent statement which was presented at the beginning of the study. The initial sample consisted of $N=316$ participants. From this initial sample, $n=33$ individuals had to be excluded because they only participated in the first of two obligatory data collection modules (online survey and supervised group survey; see below). The final sample included $N=283$ students from all fields, with an age range from 18 to 46 years and a mean age of $23.53(S D=3.25)$ years. Eighty-six percent $(n=$ 228) of the participants were females. 


\section{Materials and Procedure}

Data were collected at two different times to prevent symptoms of exhaustion. First, participants completed an online survey (about $30 \mathrm{~min}$ ). In the two weeks following the completion of this survey, they additionally participated in a supervised group survey. Attending the group survey was not possible without participating in the online survey and all participants from the online survey were allowed to participate in the group survey. The supervised group survey enabled us to ensure that participants were uninterruptedly exposed to a depicted health scenario they had to empathize with. Thus, they were not able to distract themselves from the scenario ( this may well have happened in an online setting). There were no inclusion or exclusion criteria following the online survey for participants to take part in the supervised group survey. Compensation for participation in both sessions was $30 €$ in total (approx. $34 \$$ ).

During the online part, approach and avoidance motivation were assessed by means of the 20 item-short version of the Action Regulation Emotion Systems questionnaire (ARES-K; Hartig and Moosbrugger 2003). The questionnaire measures approach and avoidance motivation on two scales (ten items per scale). Statements were captured on a 4-point Likert scale $(1=$ strong disagreement to $4=$ strong agreement $)$. For both scales, internal consistency was found to be good to very good, with Cronbach's $\alpha=.90$ for the BIS-scale and $\alpha=.83$ for the BAS-scale. Emotion regulation ability was determined by the mean of the three 12 item-scales "anxiety regulation", "stress regulation" and "regulation of positive emotions" from the Self-report for the Assessment of Emotion-Specific Regulation Skills (SEK-ES; Ebert et al. (2013). The items are scored on a 5 -point Likert scale $(1=$ not all, $5=$ always $)$. Internal consistency of the merged scales version (36 items in total) was very good (Cronbach's $\alpha=.92$ ). In the second part of the study, among other questionnaires related to a different study, questionnaires assessing the remaining variables were applied. Self-efficacy was measured by the Self-Efficacy Scale for Information Behavior (SES-IB; Behm 2015) with a slightly adjusted instruction relating to a health context. The questionnaire consists of 16 items that are scored on a 5-point Likert scale $(1=$ does not apply at all, $5=$ fully applies $)$. Internal consistency of this scale was very good (Cronbach's $\alpha=.91$ ). To assess HIS emotion and problem focus, the Goals Associated with Health Information Seeking questionnaire (GAINS; Chasiotis, Wedderhoff, Rosman, \& Mayer, 2019) was used. In this questionnaire, the scales 'Reassurance' and 'Hope' constitute an emotion focus in HIS (eight items in total), whereas the scales 'Understanding' and 'Action Planning' measure two different aspects of a problem focus in HIS (eight items in total). Items are scored on a 5-point Likert scale $(1=$ does not apply at all, $5=$ fully applies $)$. Internal consistency for both merged scales was good
(Cronbach's $\alpha=.88$ for emotion focus and Cronbach's $\alpha=.87$ for problem focus). Intended comprehensiveness of HIS was assessed by the six item-Thoroughness of Search (TOS) scale developed by Heinström (2002). The items are scored on a 5-point Likert scale $(1=$ does not apply at all, $5=$ totally applies). Internal consistency was satisfactory (Cronbach's $\alpha=.76$ ).

Before completing the questionnaires, participants were instructed to imagine themselves being in a specific healththreatening situation (experiencing chest pain) and answer the questionnaires accordingly. This was done in order to put the participants in a state of perceiving a threat to their own health, which may elicit more realistic self-reports of intended information behavior. We chose chest pain as a scenario topic because it is a symptom possibly indicating a lifethreatening cause, and because the annual prevalence of chest pain is very high in the general population as well as, specifically, in younger individuals (18-29 years; Eslick et al. 2003; Fass and Achem 2011). We expected that this would aid our participants in relating to the depicted scenario. As manipulation check, participants rated the perceived threat of the scenario on a scale from 0 (no threat) to 100 (maximum possible threat; see also Chasiotis et al. 2019). According to this scale, perceived threat was moderate $(M=50.12, S D=23.77)$. It can therefore be assumed that the test persons were aware of a (fictitious) health threat when answering the questions. All variables were normally distributed. Descriptive statistics of the model variables including their respective correlations are presented in Table 1.

\section{Analysis}

In order to test the hypothesized relationships, Structural Equation Modeling (SEM) was conducted via R using the lavaan package by Rosseel (2012). We chose SEM because it allows to simultaneously analyze the model paths and test the goodness of fit of the whole model. To test the specific hypotheses about effects within the model, specific model parameters can be consulted. For direct effects from one latent factor to another, the corresponding path coefficient is regarded. For indirect effects, new parameters are defined as the product of the involved path coefficients. Maximum likelihood estimation was used to estimate the model parameters.

Figure 1 depicts the linear structural model which we developed in accordance with theoretical considerations and to test our hypotheses. Besides the specific effects reflected in our hypotheses, we included two additional paths. First, we allowed an effect from approach motivation on problem focus (path $\gamma_{1}$ ) to test if the indirect effect via emotion regulation and self-efficacy postulated in Hypothesis 2 is indeed the only possible way in which approach motivation impacts HIS. Second, we included an effect from HIS self-efficacy on intended comprehensiveness of search (path $\gamma_{7}$ ) to analyze 
Table 1 Correlations Between Model Variables (Cronbach's Alpha in Italics)

\begin{tabular}{llllllll}
\hline Model variable & 1 & 2 & 3 & 4 & 5 & 6 & 7 \\
\hline 1 Approach Motivation & .83 & & & & & & \\
2 Avoidance Motivation & $-.21^{* *}$ & .90 & & & & & \\
3 Emotion Regulation & $.47^{* *}$ & $-.41^{* *}$ & .92 & & & & \\
4 Self-Efficacy & .11 & $-.14^{*}$ & $.31^{* *}$ & .79 & & & \\
5 Problem Focus & $.13^{*}$ & .02 & .08 & $.19^{* *}$ & .87 & & \\
6 Emotion Focus & .06 & $.15^{*}$ & .02 & .06 & $.42^{* *}$ & .90 & \\
7 Comprehensiveness of Search & .08 & -.08 & .09 & $.17^{* *}$ & $.43^{* *}$ & $.21^{* *}$ & .76 \\
$M$ & 3.28 & 2.64 & 3.74 & 3.61 & 8.08 & 6.68 & 3.55 \\
$S D$ & 0.44 & 0.61 & 0.48 & 0.49 & 1.43 & 1.68 & 0.64 \\
\hline
\end{tabular}

$* p<.05 . * * p<.01$

Values between .10 and .30 may be significant but represent small effects (Cohen 1992) the importance of this effect in the presence of an indirect effect of self-efficacy via problem focus as postulated in Hypothesis 2. Furthermore, in accordance with theory, we allowed covariations between approach and avoidance motivation (Hartig and Moosbrugger 2003) and between HIS problem and emotion focus (Chasiotis et al., 2019). We did not include the direct effects from approach and avoidance motivation on intended comprehensiveness of search in addition to the postulated indirect effects that we derived from theory (see Hypothesis 2). This was because there is not enough theoretical support for the corresponding assumption of direct effects operating independently from all other included variables (e.g., coping focus). ${ }^{1}$ Additionally, since there was a certain amount of variability in how threatening participants perceived the scenario, we controlled for the degree of perceived threat in an additional model. However, this did not impact path coefficients or general model fit. For reasons of parsimony, we therefore did not include perceived threat as a covariate in our final model.

\section{Results}

Results can be found in Table 2 and Fig. 1. The factor loadings of the indicator variables on their respective latent variables were all significant $(p<.001)$. This supports the assumption that the observed variables adequately represent their associated constructs. The proportion of variance in intended comprehensiveness of search explained by the model was $\mathrm{R}^{2}=.325$. The overall fit of the estimated model was good $\left(\chi^{2}=332.58, d f=218, p<.001, \mathrm{CFI}=.96, \mathrm{TLI}=.96\right.$, SRMR $=.056, \mathrm{RMSEA}=.043$; see Schermelleh-Engel et al. 2003). Therefore, a further investigation of the model parameters is valid with respect to the formulated hypotheses.

\footnotetext{
${ }^{1}$ In accordance with this, exploratory analyses of these direct paths yielded no significant effects.
}

In accordance with Hypothesis 1, avoidance motivation had a negative effect on emotion regulation $\left(\gamma_{3}=-.44, p\right.$ $<.05$ ), whereas approach motivation had a positive effect on emotion regulation $\left(\gamma_{2}=.36, p<.05\right)$.

The inspection of the indirect effects shows that approach motivation ( $\left.\mathrm{IDE}_{1}=.01, p<.05\right)$, as well as avoidance motivation $\left(\mathrm{IDE}_{2}=-.01, p<.05\right)$ had a small, albeit significant indirect effect on intended comprehensiveness of search, mediated by emotion regulation, HIS self-efficacy, and HIS problem focus. The direct effect of approach motivation on problem focus was not significant, indicating that the indirect effect of approach motivation on intended comprehensiveness of search via emotion regulation and self-efficacy is the only one with a significant impact. Thus, Hypothesis 2 is also supported.

In accordance with Hypothesis 3, the regression weight of the direct path from avoidance motivation on emotion focus in HIS was significant $\left(\gamma_{4}=.16, p<.05\right)$. Our exploratory analysis concerning the path from HIS emotion focus on intended

Table 2 Fit Indices and Hypothesized Indirect Effects of the Structural Model

\begin{tabular}{ll}
\hline Fit index/IDE & Coefficient \\
\hline$\chi^{2}$ & 332.58 \\
$d f$ & 218 \\
$\chi^{2} / d f$ & 1.53 \\
$\mathrm{CFI}$ & .96 \\
$\mathrm{TLI}$ & .96 \\
SRMR & .056 \\
RMSEA (90\% CI) & $.043(.034-.052)$ \\
Approach motivation $\rightarrow$ & $.01 *$ \\
Comprehensiveness (IDE $)^{\mathrm{a}}$ & \\
Avoidance motivation $\rightarrow$ & $-.01 *$ \\
Comprehensiveness (IDE & \\
\hline a & \\
\hline ing (HIS) self-efficacy and intended problem focus of HIS \\
$* p<.05$
\end{tabular}


comprehensiveness of search showed no significant effect $\left(\gamma_{10}=.03, p=.72\right)$.

\section{Discussion}

In this study, we included motivational variables together with emotion regulation and HIS self-efficacy in a model with coping foci and intended behavior related to a specific form of engagement coping, health information seeking. The model fit was good, and all our three hypotheses were supported. Approach motivation had a positive effect on emotion regulation, whereas avoidance motivation had a negative effect on emotion regulation (Hypothesis 1). There also was a significant indirect effect from approach and avoidance motivation (positive and negative, respectively) on intended comprehensiveness of search via emotion regulation, HIS self-efficacy and problem focus (Hypothesis 2). For approach motivation, there was no significant direct effect on problem focus (path $\gamma_{1}$ in Fig. 1) and thus, no indirect effect on intended comprehensiveness of search mediated by problem focus only (paths $\gamma_{1}$ and $\gamma_{9}$ in Fig. 1). This suggests that associations between approach motivation and engagement coping, as observed in other studies (e.g., Litman 2006), may be the result of intermediary effects of emotion regulation and self-efficacy, which seems to be true at least for HIS. The perceived ability to emotionally deal with a threat (emotion regulation) and the conviction that one is able to deal with potentially threatening information (HIS self-efficacy) may increase the perceived controllability of a situation. This, in turn, may enable the adoption of a problem coping focus (Folkman and Lazarus 1980; Rothermund 2011). Furthermore, the adaptation of a problem coping focus, meaning the intention to directly tackle and overcome the health problem, facilitates an extensive and balanced search. This may be because the results of such an inquiry are supposedly most useful to aid in dealing with the problem (Johnson and Knobloch-Westerwick 2017).

As for avoidance motivation, consistent with our third hypothesis, there was a direct effect on emotion focus which in turn had no effect on intended comprehensiveness of search. This unique effect of avoidance motivation on an emotion focus in HIS might be explained by the high sensitivity to threat and threat-related emotions captured by avoidance motivation (Carver 2005) which is particularly strong when confronted with a (potentially existential) health threat. For this reason, we suppose that (higher) avoidance motivation demands additional, external means which complement the individual emotion regulation capacities to downregulate these threat-related emotions. In case of a health threat, HIS serves this purpose: health information is accessed to deal with one's unpleasant emotions and, for example, to find reassuring explanations for a health problem.

The absent effect from emotion coping focus on intended comprehensiveness of search suggests that a careful and thorough search is not imperative to fulfil this purpose. Sometimes, the first reassuring information one finds might be enough, and other times, one piece of information, contrary to expectations, might have increased experienced distress. In this case, the search would have to go on and might come forth more thorough. However, it is not suitable to imply that there is no effect only because none was found in our study. Therefore, these considerations are merely speculative. Nonetheless, our findings regarding the differential effects of coping foci are in line with past results on the impact of different coping foci on information behavior (e.g., Johnson and Knobloch-Westerwick 2017; Kalichman et al. 2006; Van der Velde and van der Pligt 1991).

Our study corroborates earlier research on the impact of personality dispositions on various kinds of health behavior (e.g., Booth-Kewley and Vickers Jr 1994; Friedman 2000; Hampson et al. 2006), as well as research more specifically focusing on the impact of motivational and affective dispositions on health information behavior (e.g., Gerend and Shepherd 2007; Hastall and Wagner 2017; Hevey and Dolan 2014; Lalot et al. 2018; Sherman et al. 2006). Therefore, with regard to clinical practice, our findings imply the necessity to anticipate divergent health information seeking behaviors and preferences between patients even when they are in the same situation (e.g., before cancer treatment; Eheman et al. 2009). Depending on motivational dispositions, emotion regulation abilities and HIS self-efficacy, health information seeking serves different coping foci and varies in its comprehensiveness. Especially individuals that are prone to experience anxiety (i.e., highly avoidance-motivated) and have emotion regulation difficulties may need support in dealing with their unpleasant feelings to enable a more problem-focused and thus, more comprehensive and balanced search (see also Van't Riet and Ruiter 2013). This is because an emotion focused search may exhibit certain problems, e.g., preferring the reassuring potential of a source over its quality. Avoidance of threatening but accurate information (Litman and Lunsford 2010), or "getting astray" in the search because a satisfying level of reassurance is never achieved, are additional potential problems of an emotion focused search. Thus, training especially avoidance-oriented individuals in their emotion regulation abilities and supporting them in dealing with feelings of stress and anxiety might provide them with the necessary means to cope with a health threat in a more problem focused way. Furthermore, health information providers (e.g., clinical practitioners, patient advisory groups) should be aware that there are interindividually differing approaches to health information. Hence, in an effort to prevent dysfunctional information behavior, they might try to supply a body of balanced and comprehensive information, while, at the same time, keeping in mind that patients have differing emotional needs that need to be addressed.

In this line of research, further analyses of the implications of our model might be fruitful. By observing actual information behavior, differences between individuals who tend to be more problem focused and/or emotion focused in their 
information search could be assessed. One possible research question could be if individuals with an emotion focused coping style are more vulnerable to an unfounded escalation of concerns about common symptoms based on their review of search results. This is also known as cyberchondria (White \& Horvitz, 2009) and is becoming a growing concern among healthcare practitioners.

Another starting point for future research would be the analysis of further influential factors on intended comprehensiveness of search in the context of a health threat. Despite our model explaining about a third of the variance in our outcome variable (which constitutes a substantial proportion), the question as to the nature of other potentially relevant explanatory variables arises. In line with this, it can be assumed that situational and dispositional factors would interact in unique ways that warrant further scrutiny. For example, according to the literature, preexisting beliefs and/or perceived severity of the health threat seem plausible factors that could meaningfully complement our model (Chang et al. 2012; McKinley and Ruppel 2014). There are some indications in the literature that the latter may interact with avoidance motivation. For example, Nestler and Egloff (2010) found that the degree of threat in a health message moderated the effect of dispositional avoidance on attitudes towards the health information and intentions regarding health promoting behavior. Furthermore, transient and highly situational specific factors like one's current mood may exhibit significant effects on search comprehensiveness in the presence of a health threat (Das 2012).

\section{Limitations}

We concede that the conclusions derived from our analysis are somewhat limited, because we exclusively assessed intended information behavior. This is in line with many theoretical models of information seeking featuring behavioral intention as outcome variable (see Kahlor 2010, for a prominent example). However, to be able to extend our conclusions to actual behavior, follow up studies should focus on behavioral outcomes, such as, for example, direct observation of information behavior during a specific search task.

Furthermore, the cross-sectional design of the present study only allows assumptions about the associations between emotion and problem focus. However, by implementing a longitudinal design with multiple points of assessment within a search process, the potential variability and prioritization of the coping foci and the corresponding seeking behavior could be analyzed. For example, as Carver and Connor-Smith (2010) discuss, it is conceivable that over time, the focus may shift from initially calming oneself through information to gaining a deeper insight into the problem itself.

A further limitation concerns the generalizability of our findings. Our sample consisted of university students and thus, participants were above average young and educated, predominantly female, and most likely rather healthy. In order to address this issue, additional studies drawing on broader samples should be conducted. Furthermore, though we aimed at approximating a 'real life-health problem situation' through confronting our study participants with a health problem scenario, real patient samples are of particular interest to further validate our theoretical model.

\section{Conclusion}

We showed that approach and avoidance tendencies and emotion regulation abilities together constitute a motivationalemotional framework of coping via HIS. We demonstrated unique courses of effects between these variables and therefore, we provide new insights regarding the impact of dispositional motivational and emotional variables on engagement coping. These insights usefully complement classic theories dealing with information seeking styles in the face of a threat which essentially differentiate between actively seeking and completely avoiding (health) information (e.g., monitoring vs. blunting, Miller 1987). Our approach enables the analysis of interindividual differences within the former, i.e. of the varying comprehensiveness of an active search for health information. Thus, with this study, we provide a fruitful starting point for further research focusing on the interplay of motivational dispositions, emotion regulation abilities, and coping behavior.

\section{Compliance with Ethical Standards}

Ethical Approval All procedures performed in studies involving human participants were in accordance with the ethical standards of the institutional research committee and with the 1964 Helsinki declaration and its later amendments or comparable ethical standards.

Conflict of Interest The authors declare that the research was conducted in the absence of any commercial or financial relationships that could be construed as a potential conflict of interest.

Open Access This article is distributed under the terms of the Creative Commons Attribution 4.0 International License (http:// creativecommons.org/licenses/by/4.0/), which permits unrestricted use, distribution, and reproduction in any medium, provided you give appropriate credit to the original author(s) and the source, provide a link to the Creative Commons license, and indicate if changes were made.

\section{References}

Baumann, N., \& Kuhl, J. (2002). Intuition, affect, and personality: Unconscious coherence judgments and self-regulation of negative affect. Journal of Personality and Social Psychology, 83(5), 1213-1223.

Behm, T. (2015). Informationskompetenz und Selbstregulation: Zur Relevanz bereichsspezifischer Selbstwirksamkeitsüberzeugungen [information competence and self-regulation: The relevance of domain-specific self-efficacy]. In A.-K. Mayer (Ed.), 
Informationskompetenz im Hochschulkontext [information competence in the higher education context.]: Interdisziplinäre Forschungsperspektiven [interdisciplinary research perspectives] (pp. 151-162). Lengerich: Pabst Science Publishers.

Berkman, N. D., Sheridan, S. L., Donahue, K. E., Halpern, D. J., Viera, A., Crotty, K., et al. (2011). Health literacy interventions and outcomes: An updated systematic review. Evidence Report/Technology Assessment, 199(1), 1-941.

Blouin-Hudon, E.-M. C., Gaudreau, P., \& Gareau, A. (2016). Coping as a building mechanism to explain the unique association of affect and goal motivation with changes in affective states. Anxiety, Stress, \& Coping, 29(5), 519-532.

Booth-Kewley, S., \& Vickers Jr., R. R. (1994). Associations between major domains of personality and health behavior. Journal of Personality, 62(3), 281-298.

Braniecka, A., Trzebińska, E., Dowgiert, A., \& Wytykowska, A. (2014). Mixed emotions and coping: The benefits of secondary emotions. PLoS One, 9(8), e103940.

Byrne, D. (1961). The repression-sensitization scale: Rationale, reliability, and validity. Journal of Personality, 29(3), 334-349.

Carver, C. S. (2005). Impulse and constraint: Perspectives from personality psychology, convergence with theory in other areas, and potential for integration. Personality and Social Psychology Review, 9(4), 312-333.

Carver, C. S., \& Connor-Smith, J. (2010). Personality and coping. Annual Review of Psychology, 61(1), 679-704.

Chang, D.-S., Kang, O.-S., Kim, H.-H., Kim, H.-S., Lee, H., Park, H.-J., et al. (2012). Pre-existing beliefs and expectations influence judgments of novel health information. Journal of Health Psychology, 17(5), 753-763.

Chasiotis, A., Wedderhoff, O., Rosman, T., \& Mayer, A.-K. (2019). Why do we want health information? The goals associated with health information seeking questionnaire (GAINS). Psychology \& Health. https://doi.org/10.1080/08870446.2019.1644336.

Cohen, J. (1992). A power primer. Psychological Bulletin, 112(1), 155159.

Das, E. (2012). Rethinking the role of affect in health communication. European Health Psychologist, 14(2), 27-31.

Das, E., \& Fennis, B. M. (2008). In the mood to face the facts: When a positive mood promotes systematic processing of self-threatening information. Motivation and Emotion, 32(3), 221-230.

Dennis, T. A. (2007). Interactions between emotion regulation strategies and affective style: Implications for trait anxiety versus depressed mood. Motivation and Emotion, 31(3), 200-207.

Derakshan, N., Eysenck, M. W., \& Myers, L. B. (2007). Emotional information processing in repressors: The vigilance-avoidance theory. Cognition and Emotion, 21(8), 1585-1614.

Ebert, D. D., Christ, O., \& Berking, M. (2013). Entwicklung und Validierung eines Fragebogens zur emotionsspezifischen Selbsteinschätzung emotionaler Kompetenzen (SEK-ES) [development and validation of a emotion-specific self-report measurement of emotional competences]. Diagnostica, 59(1), 17-32.

Eheman, C. R., Berkowitz, Z., Lee, J., Mohile, S., Purnell, J., Marie Rodriguez, E., et al. (2009). Information-seeking styles among cancer patients before and after treatment by demographics and use of information sources. Journal of Health Communication, 14(5), 487502

Elliot, A. J., \& Church, M. A. (1997). A hierarchical model of approach and avoidance achievement motivation. Journal of Personality and Social Psychology, 72(1), 218-232.

Eslick, G. D., Jones, M. P., \& Talley, N. J. (2003). Non-cardiac chest pain: Prevalence, risk factors, impact and consulting- a population-based study. Alimentary Pharmacology \& Therapeutics, 17(9), 11151124.
Fass, R., \& Achem, S. R. (2011). Noncardiac chest pain: Epidemiology, natural course and pathogenesis. Journal of Neurogastroenterology and Motility, 17(2), 110-123.

Feil, J., \& Hasking, P. (2008). The relationship between personality, coping strategies and alcohol use. Addiction Research \& Theory, 16(5), 526-537.

Folkman, S. (2010). Stress, coping, and hope. Psycho-Oncology, 19(9), 901-908.

Folkman, S., \& Lazarus, R. S. (1980). An analysis of coping in a middleaged community sample. Journal of Health and Social Behavior, 21(3), 219-239.

Friedman, H. S. (2000). Long-term relations of personality and health: Dynamisms, mechanisms, tropisms. Journal of Personality, 68(6), 1089-1107.

Gerend, M. A., \& Shepherd, J. E. (2007). Using message framing to promote acceptance of the human papillomavirus vaccine. Health Psychology, 26(6), 745-752.

Hampson, S. E., Andrews, J. A., Barckley, M., Lichtenstein, E., \& Lee, M. E. (2006). Personality traits, perceived risk, and risk-reduction behaviors: A further study of smoking and radon. Health Psychology, 25(4), 530.

Hart, W., Albarracín, D., Eagly, A. H., Brechan, I., Lindberg, M. J., \& Merrill, L. (2009). Feeling validated versus being correct: A metaanalysis of selective exposure to information. Psychological Bulletin, 135(4), 555-588.

Hartig, J., \& Moosbrugger, H. (2003). Die “ARES-Skalen” zur Erfassung der individuellen BIS-und BAS-Sensitivität [the ARES-scales as a measurement of individual BIS- and BAS-sensitivity: Development of a long and a short questionnaire version]. Zeitschrift Für Differentielle Und Diagnostische Psychologie, 24(4), 293-310.

Hasking, P. A. (2006). Reinforcement sensitivity, coping, disordered eating and drinking behaviour in adolescents. Personality and Individual Differences, 40(4), 677-688.

Hastall, M. R., \& Wagner, A. J. M. (2017). Enhancing selective exposure to health messages and health intentions. Journal of Media Psychology, 30. https://doi.org/10.1027/1864-1105/a000197.

Heinström, J. (2002). Fast surfers, broad scanners and deep divers: Personality and information-seeking behaviour. Abo: Abo Akademi University Press.

Hevey, D., \& Dolan, M. (2014). Approach/avoidance motivation, message framing and skin cancer prevention: A test of the congruency hypothesis. Journal of Health Psychology, 19(8), 1003-1012.

Hundt, N. E., Williams, A. M., Mendelson, J., \& Nelson-Gray, R. O. (2013). Coping mediates relationships between reinforcement sensitivity and symptoms of psychopathology. Personality and Individual Differences, 54(6), 726-731.

Johnson, B. K., \& Knobloch-Westerwick, S. (2017). Steer clear or get ready: How coping styles moderate the effect of informational utility. Journal of Broadcasting \& Electronic Media, 61(2), 332-350.

Kahlor, L. (2010). PRISM: A planned risk information seeking model. Health Communication, 25(4), 345-356.

Kalichman, S. C., Cherry, C., Cain, D., Weinhardt, L. S., Benotsch, E., Pope, H., \& Kalichman, M. (2006). Health information on the internet and people living with HIV/AIDS: Information evaluation and coping styles. Health Psychology, 25(2), 205-210.

Kiesler, D. J., \& Auerbach, S. M. (2006). Optimal matches of patient preferences for information, decision-making and interpersonal behavior: Evidence, models and interventions. Patient Education and Counseling, 61(3), 319-341.

Koole, S. L. (2009). The psychology of emotion regulation: An integrative review. Cognition and Emotion, 23(1), 4-41.

Krohne, H. W., \& Hock, M. (2011). Anxiety, coping strategies, and the processing of threatening information: Investigations with cognitive-experimental paradigms. Personality and Individual Differences, 50(7), 916-925. 
Kuhl, J., \& Koole, S. L. (2008). The functional architecture of approach and avoidance motivation. In A. J. Elliot (Ed.), Handbook of approach and avoidance motivation (pp. 535-555). New York: Taylor \& Francis.

Lalot, F., Quiamzade, A., \& Zerhouni, O. (2018). Regulatory focus and self-determination motives interact to predict students' nutritionhabit intentions. Journal of Experimental Psychology: Applied. Advance online publication. https://doi.org/10.1037/xap0000196

Lazarus, R. S. (2006). Emotions and interpersonal relationships: Toward a person-centered conceptualization of emotions and coping. Journal of Personality, 74(1), 9-46.

Litman, J. A. (2006). The COPE inventory: Dimensionality and relationships with approach-and avoidance-motives and positive and negative traits. Personality and Individual Differences, 41(2), 273-284.

Litman, J. A., \& Lunsford, G. D. (2010). Incurious motives to seek information about potential threats. European Journal of Personality, 24(8), 639-655.

McKinley, C. J., \& Ruppel, E. K. (2014). Exploring how perceived threat and self-efficacy contribute to college students' use and perceptions of online mental health resources. Computers in Human Behavior, 34, 101-109. https://doi.org/10.1016/j.chb.2014.01.038.

Miller, S. M. (1987). Monitoring and blunting: Validation of a questionnaire to assess styles of information seeking under threat. Journal of Personality and Social Psychology, 52(2), 345-353.

Nestler, S., \& Egloff, B. (2010). When scary messages backfire: Influence of dispositional cognitive avoidance on the effectiveness of threat communications. Journal of Research in Personality, 44(1), 137-141.

Pocnet, C., Dupuis, M., Congard, A., \& Jopp, D. (2017). Personality and its links to quality of life: Mediating effects of emotion regulation and self-efficacy beliefs. Motivation and Emotion, 41(2), 196-208.

Rosseel, Y. (2012). Lavaan: An R package for structural equation modeling. Journal of Statistical Software, 48(2), 1-36.

Rothermund, K. (2011). Counter-regulation and control-dependency: Affective processing biases in the service of action regulation. Social Psychology, 42(1), 56-66.

Schermelleh-Engel, K., Moosbrugger, H., \& Müller, H. (2003). Evaluating the fit of structural equation models: Tests of significance and descriptive goodness-of-fit measures. Methods of Psychological Research Online, 8(2), 23-74.
Schutz, P. A., Benson, J., \& Decuir-Gunby, J. T. (2008). Approach/ avoidance motives, test emotions, and emotional regulation related to testing. Anxiety, Stress, \& Coping, 21(3), 263-281.

Sherman, D. K., Mann, T., \& Updegraff, J. A. (2006). Approach/ avoidance motivation, message framing, and health behavior: Understanding the congruency effect. Motivation and Emotion, 30(2), 164-168.

Shiloh, S., \& Orgler-Shoob, M. (2006). Monitoring: A dual-function coping style. Journal of Personality, 74(2), 457-478.

Showers, C. J., \& Boyce, K. L. (2008). Secrets of resilience: Approaching negative self-aspects without aversion. In A. J. Elliot (Ed.), Handbook of approach and avoidance motivation (pp. 521-534). New York: Taylor \& Francis.

Sweeny, K., Melnyk, D., Miller, W., \& Shepperd, J. A. (2010). Information avoidance: Who, what, when, and why. Review of General Psychology, 14(4), 340-353.

Tamir, M. (2016). Why do people regulate their emotions? A taxonomy of motives in emotion regulation. Personality and Social Psychology Review, 20(3), 199-222.

Tamir, M., \& Diener, E. (2008). Well-being: Approach-avoidance goals and well-being: One size does not fit all. In A. J. Elliot (Ed.), Handbook of approach and avoidance motivation (pp. 415-428). New York: Taylor \& Francis.

Tull, M. T., Gratz, K. L., Latzman, R. D., Kimbrel, N. A., \& Lejuez, C. W. (2010). Reinforcement sensitivity theory and emotion regulation difficulties: A multimodal investigation. Personality and Individual Differences, 49(8), 989-994.

Van der Velde, F. W., \& van der Pligt, J. (1991). AIDS-related health behavior: Coping, protection motivation, and previous behavior. Journal of Behavioral Medicine, 14(5), 429-451.

Van't Riet, J., \& Ruiter, R. A. C. (2013). Defensive reactions to healthpromoting information: An overview and implications for future research. Health Psychology Review, 7, 104-136. https://doi.org/ 10.1080/17437199.2011.606782.

Publisher's note Springer Nature remains neutral with regard to jurisdictional claims in published maps and institutional affiliations. 